\title{
THE PHYSICAL BASIS OF COLOR-TECHNOLOGY.*
}

\author{
BY
}

\author{
M. LUCKIESH, \\ Physicist, Nela Research Laboratory.
}

One of the most annoying features of dyes is the extreme rarity of pure blue dyes. Nearly all blue dyes, Table VII, transmit the extreme red rays quite freely, and the scarcity of bluegreen dyes which are not dichroic makes it difficult often to find a combination which transmits only the violet rays. In extremely; high concentrations or great depths some blue dyes effectually absorb most of the extreme red rays.

In Table VIII are presented a number of spectral analyses grouped under the common name of purple for the purpose of classification. An interesting case is that of ethyl violet in gelatine, both wet and dry. After the dyed gelatine, which was flowed on clear glass, had set, and while still wet, the spectral analysis was made. The sample was then allowed to dry and another spectral analysis was made. On plotting these data a decided difference in the spectral transmission curves is seen, as indicated by the numerical data. The wet specimen is decidedly more reddish than when dry, and an actual shift in the absorption band takes place on drying. Although not definitely established, this may be explained as due to a difference in the refractive index of the solvent in the two cases. The data are corrected for reflections from the gelatine and glass surfaces.

In Table IX are presented spectral analyses of dyed gelatine filters before and after fading by exposure to solar radiation. Such data are of special interest in many cases, and it appears of interest to make a thorough study of the fading of dyes with the aid of spectral analyses. Certainly no great amount of information is available regarding the relation of the spectral character of radiation to the spectral deterioration of dyes or the relation of either of these to the chemical composition. Some work along this line is in progress. Incidentally, the testing of dyes under illuminants containing ultraviolet rays of extremely short wavelengths which are practically absent in solar radiation at the

* Concluded from page 93. July issue. 
营 趛 常 亲|

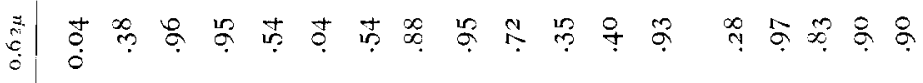
声|

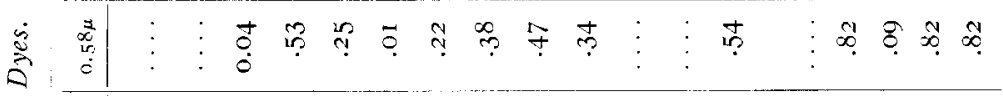

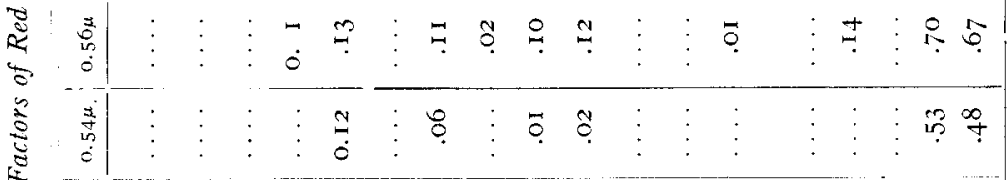

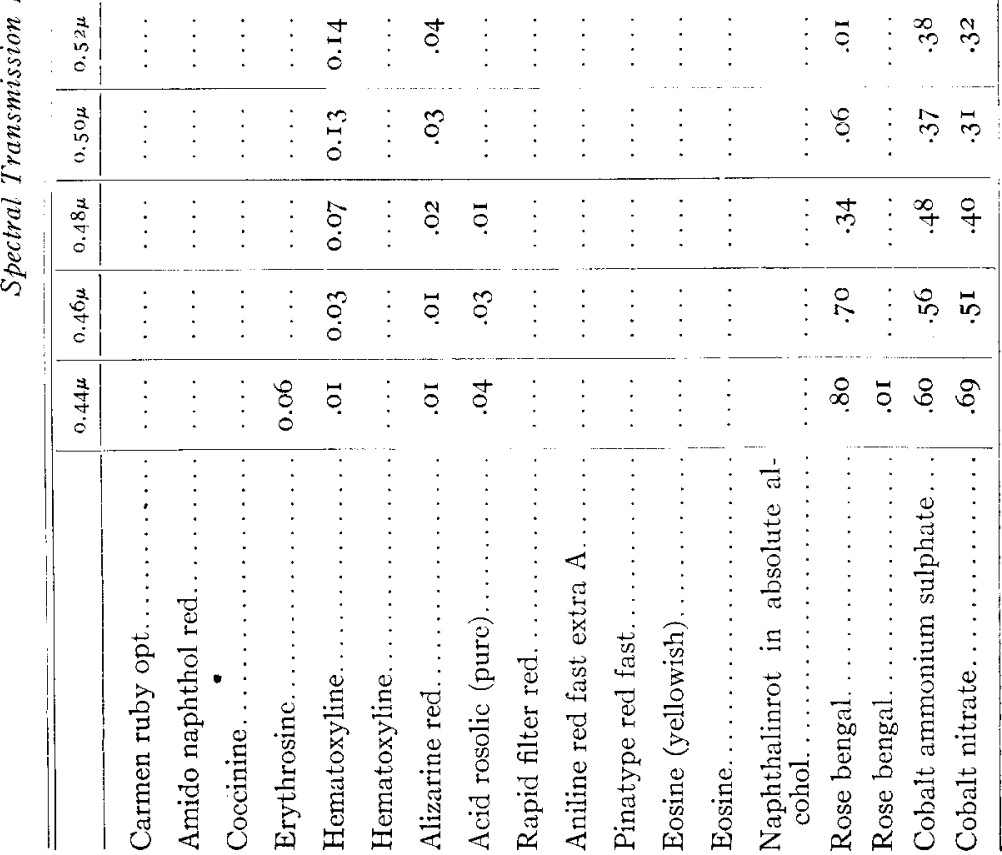




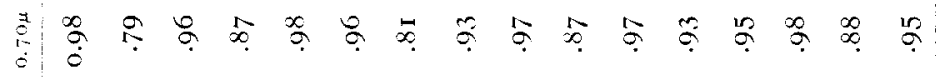

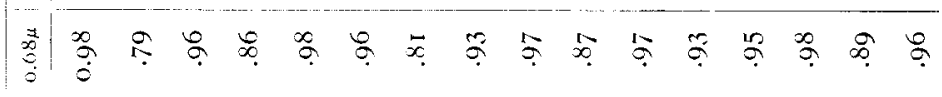

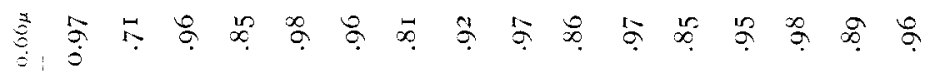

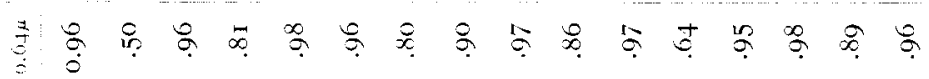

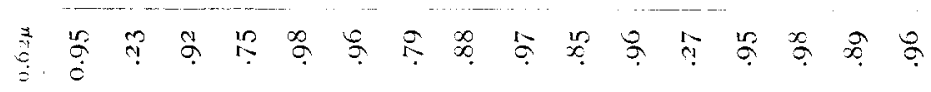

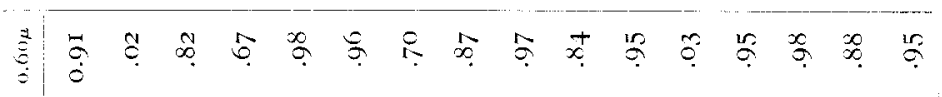

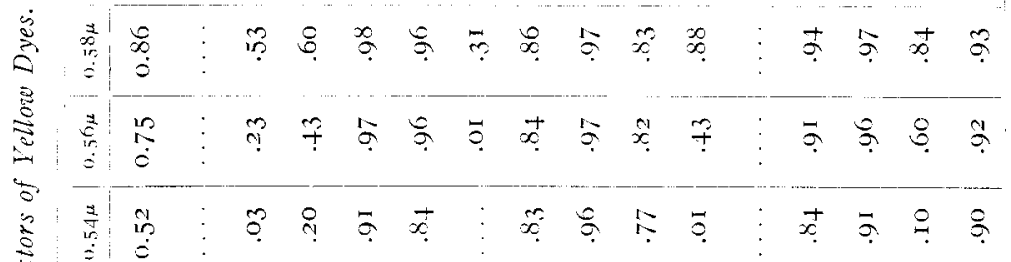

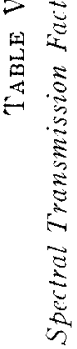

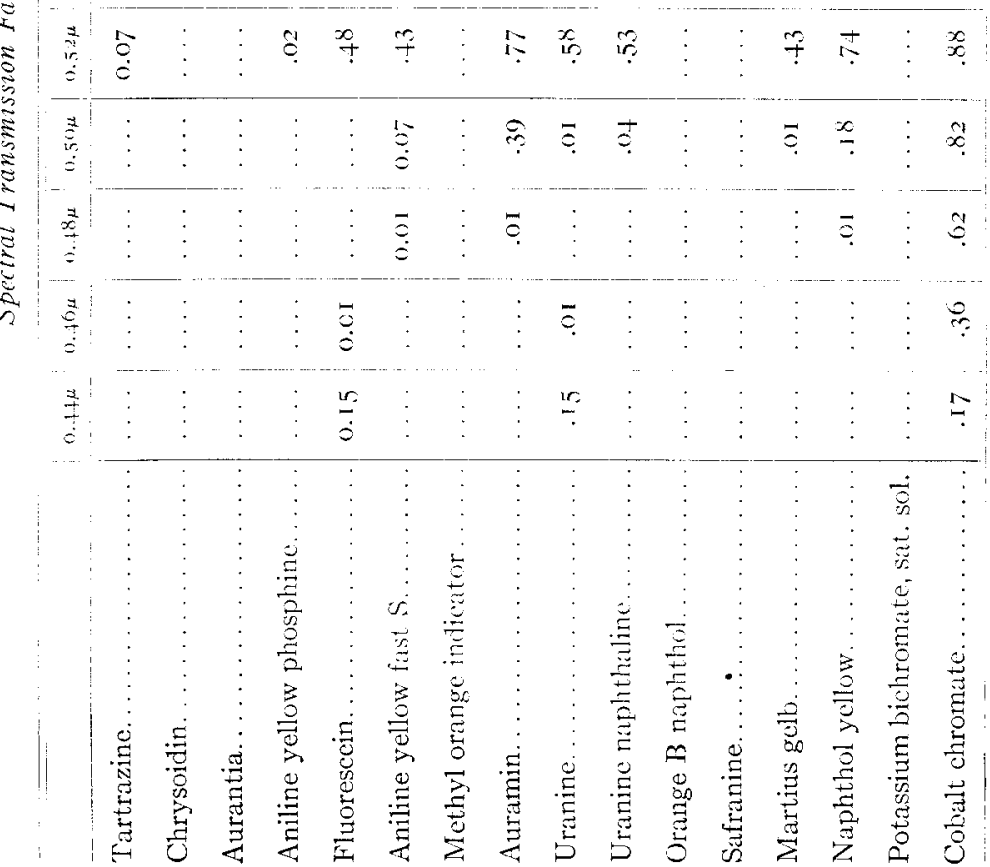




\begin{tabular}{|c|c|c|c|c|c|c|c|c|c|c|c|c|c|c|c|}
\hline 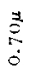 & : & $\stackrel{+}{0}$ & & $\vdots$ & ?: & $\widetilde{\Upsilon}$ & $\stackrel{\circ}{?}$ & $\stackrel{?}{?}$ & 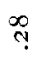 & \pm & & & 5 & $\stackrel{\sim}{a}$ & \\
\hline 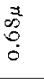 & : & $\stackrel{\ddot{y}}{\tilde{o}}$ & & $\vdots$ & $\stackrel{Y}{Y}$ & है & : & ț & $\stackrel{0}{0}$ & $\stackrel{s}{0}$ & & & & & $\stackrel{\text { ov }}{0}$ \\
\hline 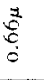 & $\vdots$ & $\begin{array}{l}\tilde{O} \\
\stackrel{0}{0}\end{array}$ & & & $\vdots$ & & & & & & $\vdots$ & & & & $\stackrel{0}{0}$ \\
\hline d & $\stackrel{\overrightarrow{0}}{\circ}$ & & & & $\vdots$ & & & . & & & & & & & 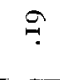 \\
\hline :ै & $\begin{array}{l}\tilde{d} \\
0 \\
0\end{array}$ & & & & $\vdots$ & : & & & & $:$ & $\tilde{o}$ & & & & ? \\
\hline : & $\begin{array}{l}\hat{0} \\
0\end{array}$ & & $\vdots$ & $\vdots$ & $\vdots$ & $\vdots$ & $\vdots$ & $\vdots$ & & & $\stackrel{t}{.}$ & & & : & คื \\
\hline 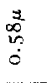 & $\stackrel{0}{\circ}$ & & $\delta$ & & $\vdots$ & $\vdots$ & $\vdots$ & $\ddot{\circ}$ & $\ddot{\circ}$ & $\vdots$ & $\underset{t}{ \pm}$ & $\stackrel{\text { ọ }}{0}$ & & : & $\hat{b}$ \\
\hline $\begin{array}{c}\vec{c} \\
\stackrel{\vec{c}}{0} \\
\stackrel{0}{0}\end{array}$ & ֶ̆ & $\vdots$ & $\because$ & $\overrightarrow{0}$ & $\vdots$ & $\vdots$ & $\vdots$ & $\stackrel{t}{\square}$ & $\stackrel{c}{0}$ & $\vdots$ & कै & oे & & : & $\stackrel{\circ}{\infty}$ \\
\hline 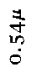 & $\stackrel{\mathscr{0}}{\ddot{c}}$ & $\stackrel{+}{\square}$ & $\widehat{\vartheta}$ & $\stackrel{\infty}{0}$ & $\vdots$ & $\vdots$ & $\vdots$ & $?$ & $\hat{0}$ & oै & $\stackrel{?}{+}$ & $\stackrel{0}{\because}$ & $\tilde{c}$ & $\vdots$ & 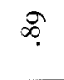 \\
\hline 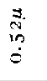 & $\stackrel{i}{i}$ & $\ddot{\vartheta}$ & $\stackrel{0}{0}$ & ְ̊ & $\ddot{c}$ & & & $\stackrel{?}{?}$ & $\cong$ & $\stackrel{\circ}{\circ}$ & ?े & 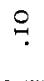 & $?$ & $\tilde{O}$ & $\tilde{\sigma}$ \\
\hline 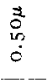 & $\begin{array}{l}\vec{y} \\
0\end{array}$ & เี้ & $R$ & $\stackrel{\varphi}{q}$ & $\stackrel{\infty}{\circ}$ & oै & $\vdots$ & in & ¿ & $\because$ & ণ̛ & $\stackrel{0}{0}$ & Fे & $\stackrel{\infty}{\longrightarrow}$ & ş \\
\hline 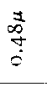 & $\begin{array}{l}1 \\
0 \\
0\end{array}$ & 8 & ț & ণి & $\stackrel{\check{y}}{\square}$ & ț & $\vdots$ & is? & $?$ & 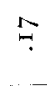 & \pm & dै. & $\ddot{q}$ & $\stackrel{?}{?}$ & $\stackrel{\infty}{\infty}$ \\
\hline $\begin{array}{l}\frac{z}{0} \\
\\
0\end{array}$ & $\begin{array}{l}0 \\
0 \\
0\end{array}$ & भे & $q)$ & \pm & $\cong$ & $\tilde{a}$ & $\vdots$ & $\overrightarrow{\dddot{y}}$ & $\overline{3}$ & $\stackrel{0}{?}$ & 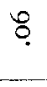 & $\vdots$ & $\stackrel{q}{q}$ & $\cong$ & మ. \\
\hline$\frac{\text { 寻 }}{3}$ & $\stackrel{0}{\circ}$ & $\stackrel{+}{0}$ & $?$ & $\%$ & $\vdots$ & . & $\vdots$ & $\hat{?}$ & $\stackrel{\infty}{\dddot{\varphi}}$ & 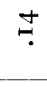 & $\ddot{0}$ & : & $\vdots$ & & $\stackrel{\curvearrowright}{\uparrow}$ \\
\hline & 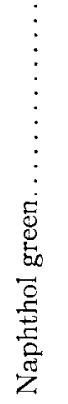 & 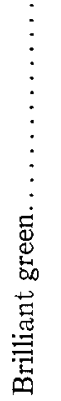 & 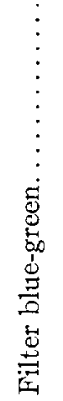 & 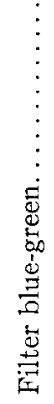 & 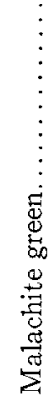 & 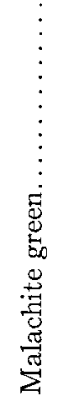 & 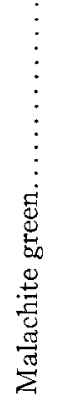 & 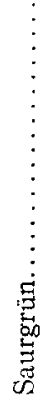 & 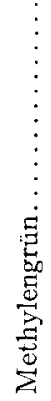 & 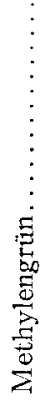 & 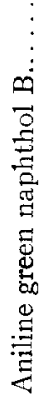 & 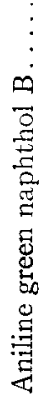 & 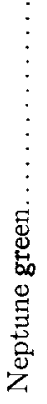 & 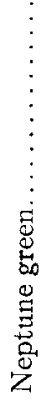 & 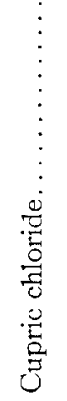 \\
\hline
\end{tabular}




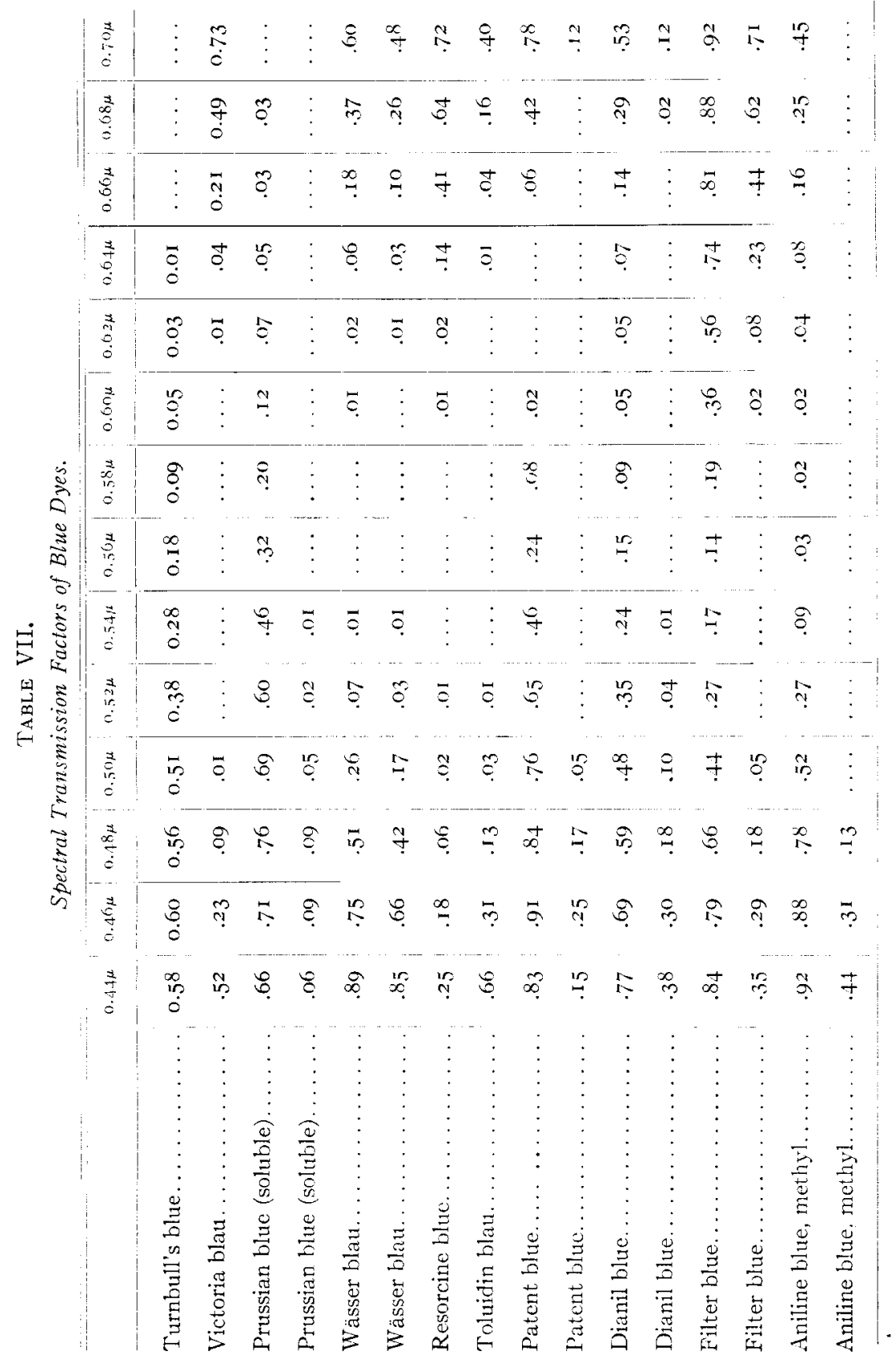









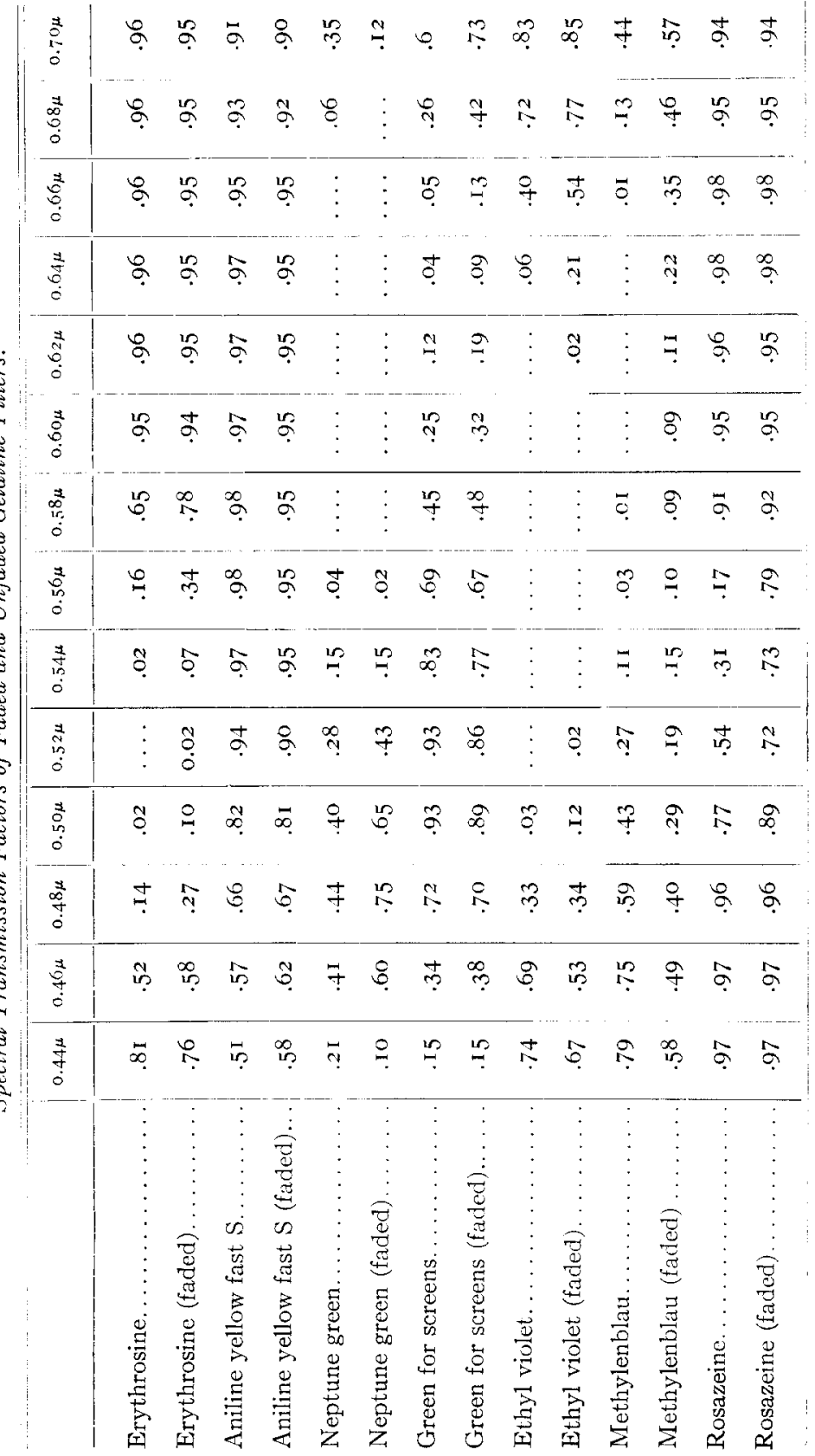


earth's surface or in artificial illuminants as commonly encountered is open to criticisnl. Spectral analysis has not been sufficiently utilized in permanency tests to warrant all the conclusions which have been drawn in this matter, although some excellent work has been done. ${ }^{\top} \mathrm{Mr}$. Mott has shown that the results with the "snow-white" flame arc in lye-fading are practically the same as those obtained in daylight.

\section{APPLICATIONS OF SPECTRAL ANALYSES OF DYES.}

The uses for spectral analyses of dyes are manifold, as in the case of any class of colored media. In general they provide a physical basis for systematic color-mixture, besides providing the necessary information for choosing dyes for many purposes. In many aspects of color-technology only the integral or subjective color is finally of interest, but the author cannot refrain from emphasizing that even in such cases an intimate knowledge of colored media and their mixtures cannot be attained without spectral analyses, and that the combination of dyes becomes systematic with such data available.

With spectrophotometric apparatus well maintained, a complete spectral analysis can be made in about an hour, although there is much room for improvement in such apparatus which will result in the saving of time. However, this is not a serious matter, because for a given coloring material only one analysis need be made, as will be shown later, to provide information for all degrees of concentration or depth of solution. The author has available hundreds of spectral analyses which, after once obtained, are a perpetual source of information.

Laws. - In order to simplify the study of coloring media, especially dyes and colored glasses, several simplifications have been made. These are based on theory and have been confirmed by experiment on a few typical specimens. In order to develop this procedure it is necessary to revert to some of the established laws. Lambert first stated that all layers of equal thickness of a transparent medium absorb equal fractions of the radiant energy which enters them. This is true for homogeneous or monochromatic radiation, but cannot be applied to the total absorption of radiant energy of many wave-lengths or of extended spectral character. It follows from Lambert's law that if the thickness of the ab-

${ }^{7}$ W. R. Mott, Trans. Amer. Eleitrochem. Soc., 1915. 
sorbing medium increases in arithmetical progression the radiation transmitted should decrease in geometrical progression.

Let $J$ be the intensity of radiation of a given wave-length entering a layer $d l$, then-

$$
-\frac{d J}{d l}=k J
$$

On integrating this we obtain:

$$
J=J_{o} e^{-k t}
$$

where $J_{o}$ is the original intensity, $J$ the intensity after traversing a thickness $d$, and $k$ is a constant depending upon the substance and upon the wave-length of the radiant energy. Various terms have been applied to this factor, such as absorption-coefficient. In logarithmic form this equation is expressed as :

$$
\log \frac{J}{J_{o}}=\log T_{\lambda}=-k_{\lambda} d \log e=-\epsilon_{\lambda} d
$$

where $T_{\lambda}$ is the transmission factor for energy of wave-length $\lambda$, and the subscripts, $\lambda$, indicate the factors which vary with the wave-length. Beer deduced the law that the absorption is the same function of the concentration of a dispersing absorbing substance as of the thickness of a single substance, which may be expressed thus:

$$
J=J_{o} A_{\lambda}^{\prime d} \text { or } T_{\lambda}=A_{\lambda}^{\text {or }} \text { or } \log T_{\lambda}=c d \log A_{\lambda}
$$

where $c$ is the concentration, $A$ is the transmission-coefficient or transmissivity, and the other symbols represent the same factors as in the foregoing equations. The validity of Beer's law has been questioned by some, and it appears that there is some doubt as to its validity in such cases as colloidal solutions. This law appears to hold when the absorbing power of a molecule is uninfluenced by the proximity of other molecules. Obviously, if any change takes place in the condition of the dispersed substance on altering the concentration the law will not hold. Incidentally there is work to be done on the validity of this law in the cases of "colloidal" glasses. Lambert's law appears to be firmly established.

In so far as the foregoing laws are valid (and it appears that this is true for all practical purposes such as described in this paper), for a given solution $\log T_{\lambda}$ is proportional to $d$, and for a given depth, or containing cell, $\log T_{\lambda}$ is proportional to $c$. By the use of coördinate paper having a logarithmic scale along one 
axis and a uniform scale along the other a great deal of interesting data can be obtained from one spectral analysis.

By means of the foregoing mathematical relations the spectral analyses of colored solutions (and colored glasses) of any thickness and concentration can be obtained from two determinations of spectral character, which often may be reduced in actual practice to a single determination. Such a method has been found exceedingly practicable in preliminary reconnoitering in search of combinations of dyes for filters, in the development of colored

FIG. I0.

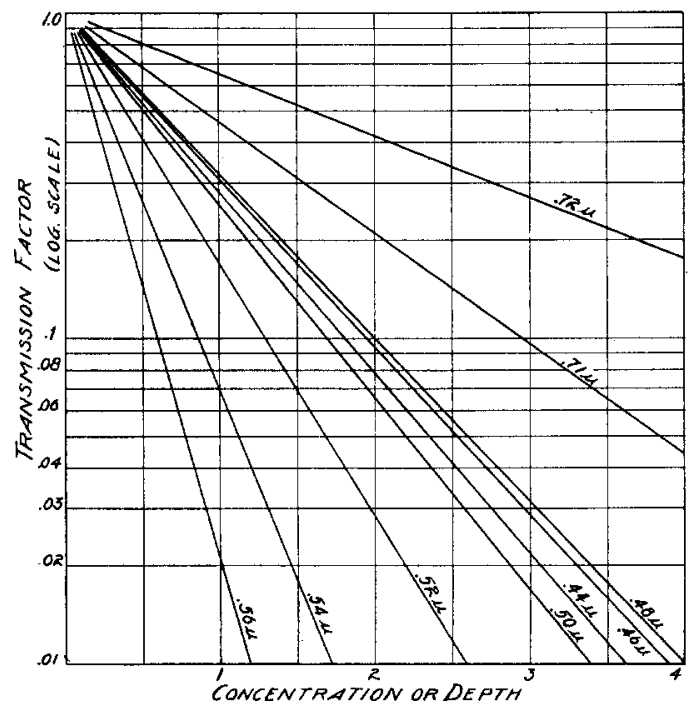

Relation between spectral transmission-factor and depth or concentration of a solution of methylengrün.

glasses, and in the study of many problems arising in colortechnology.

Some examples will suffice to illustrate the uses of this scheme in practice. Assume a solution of methylengrün of either known or unknown concentration. A cell of a known thickness is filled with the solution and a spectral analysis is made. For such a purpose a fairly low concentration or small depth is chosen, so that radiations of all wave-lengths which are of interest are appreciably transmitted. On logarithmic paper, as previously described, a plot is made, as shown in Fig. Io, the transmission factors $^{8}$ from the spectral analysis being plotted on the logarith- 
mic scale vertically above the arbitrarily selected point on the abscissa axis in this case taken as unity. The abscissæ scale may represent either concentration or depth and may be either a relative or an absolute scale. Straight lines are drawn through the points to a common point on the ordinate axis representing complete transparency or unity on this logarithmic scale. This is the common point if corrections have been made for surface reflections in the cell or from the glass surfaces in the case of a colored glass. If these corrections have not been made, the common point usually will be near 0.92 on the "transmission axis" if two surface reflections must be accounted for. Each straight line represents the relation of $\log T_{\lambda}$ and depth or concentration for a certain wave-length. By extending these lines the spectral characteristic of any depth or concentration may be read from the corresponding vertical line. If the original spectral analysis has been made with care, such a simple plot yields a vast amount of data.

Dichromatism.-Methylengrün has been chosen in Fig. Io because it also illustrates the interesting case of dichromatism so commonly exhibited by dyes. It is seen that the slope of the line for $0.72 \mu$ is less than any of the others. This is proof that the dye is a dichroic. Some lines are very steep, which indicates a large value of the absorption-coefficient for radiation of these wave-lengths. From the plot it is seen that this dye, in solutions of high concentration or of great depth, will not be green, but will be red.

Another interesting plot, of a similar nature, but including relative luminosity-values instead of transmission factors, is shown in Fig. I I for rosazeine. The spectral transmission factors for the spectral analysis used were multiplied by the visibility of radiation in each case (using Nutting's data) and plotted vertically above the point designated by unity on the concentration or depth scale. Instead of drawing straight lines representing various wave-lengths to a common point on the ordinate axis, each line is drawn to a point of this axis corresponding to the relative visibility of radiation of the particular wave-length. The

${ }^{8}$ Note.-Transmission factor is defined by the Illuminating Engineering Society as the ratio of the transmitted to the incident flux. In the present use the surface reflections are eliminated in order to make the data more useful, so that the same definition applies here for the given thickness if the body is assumed to be immersed in medium of the same refractive index. 
ordinate axis is now a logarithmic scale of relative luminosity. By extending these straight lines a graphical picture of spectral luminosity of the dye-solution is obtained for any concentration of depth. It is seen that this solution in great depth or high concentration becomes deep red, because the slopes of the lines become less with increasing wave-length after the absorption band of a weak solution or small depth is passed. Incidentally it will be noted that the slope of line $0.44 \mu$ is less than that of $0.58 \mu$, which shows that in low concentrations or in relatively small

FIG. II.

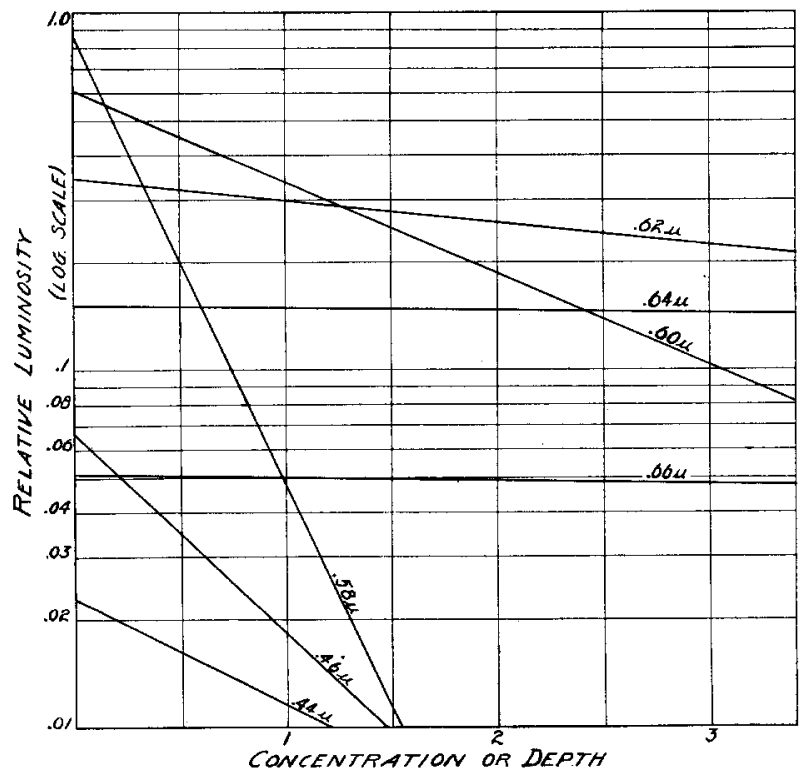

Relation between spectral luminosity and depth or concentration of a solution of rosazeine.

depths of a higher concentration the solution is purple; that is, it has an absorption band somewhere between $0.44 \mu$ and $0.60 \mu$. Only a few wave-lengths have been used for the sake of clearness.

Complete Representation of the Graphical Method.-In reality the schemes illustrated in Fig. Io and I I are only completely illustrated by means of a solid, of which, for example, Fig. Io represents a projection upon the face of the solid bounded by the logarithmic "transmission" scale and the concentration or depth scale. A model of this tri-dimensional diagram can be easily made and should be instructive. An attempt is made in 
Fig. I2 to illustrate the relations between transmission factor, wave-length, and concentration or thickness. For this purpose the spectral analysis of a thin piece of gold red glass was chosen. Many of the cross-section lines have been omitted for the sake of clearness. The scales are designated and the thickness of the specimen of gold ruby glass is assumed to be 2 units on the relative thickness scale. In plane 2, represented by the dash-dot vertical rectangle, the spectral transmission is shown in the dash-dot curve, $a_{2} b_{2} c_{2}$. For the limiting case of zero thickness this curve becomes a straight line, $T=\mathrm{I}$, which is the top edge of the foremost rectangle, plane o. Several points of the "master" curve in plane 2 were taken for the purpose of illustrating the determination of the spectral characteristic of the glass at another thickness. In this example thickness 5 units is taken and its spectral transmission is shown by the dotted curve in plane $\overline{5}$, the farthest vertical rectangle. This curve is obtained by drawing straight lines in the "wave-length" planes from the wave-lengths on the upper front scale through the points on the "master" curve in plane 2 of corresponding wave-lengths. Thus where a given straight line intersects the various thickness planes the transmission factors for that wave-length are found. For example, $b_{2}$ is a point on the "master" curve in plane 2 , and its value as read from the transmission scale is the transmission factor of this specimen of thickness, 2 units, for radiation of wave-length $0.52 \mu$. A straight line drawn through this wave-length on $T=-\mathrm{I}$ and through $b_{2}$ (always remaining in the particular wave-length plane) when prolonged intersects plane 5 at $b_{5}$, which is the transmission factor for $0.52 \mu$ for a specimen of the same glass of 5 units thickness. Other points, $a_{5}, c_{5}$, etc., are found in the same manner.

These straight lines are the same as those shown in Fig. 19; in fact, Fig. I 9 would be seen on viewing the solid, Fig. I 2, from the right-hand side. A model of this solid made of wires and painted to represent the spectral colors should be instructive.

In Fig. I I luminosity values were treated in a manner similar to the transmission values in Fig. IO. These also can be completely represented by a solid in a manner similar to that shown in Fig. 1 2, excepting that the vertical scale must represent logarithms of luminosity. In the limiting case of zero thickness the curve will not be the foremost upper horizontal line, but will be VOL. I84, No IIOO-I8 
the spectral luminosity curve of radiation and will lie in the foremost vertical plane On viewing such a solid in projection from the proper side, Fig. 20 will be seen if the same gold ruby specimen be taken as an example. It appears unnecessary to illustrate this possibility, since the general procedure should be understood from the foregoing. In case the analysis is to be made for a

FIG. 12.

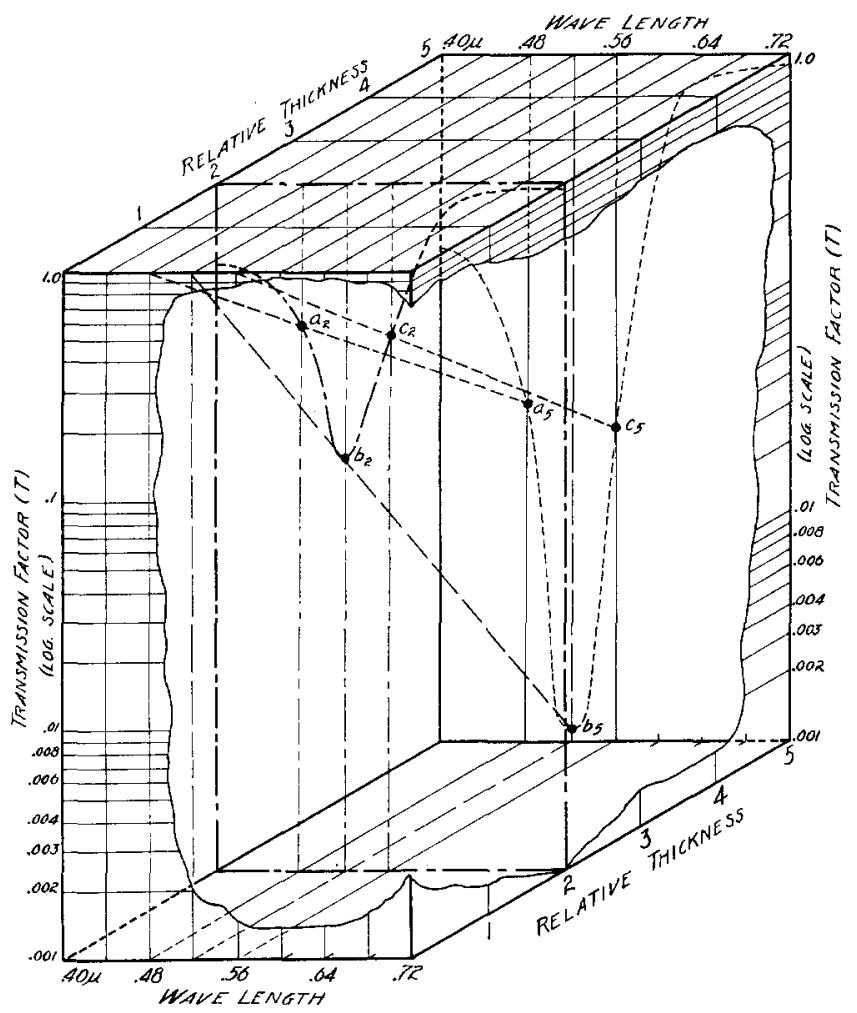

Complete relation between thickness, wave-length, and transmission-factor for a gold ruby glass.

particular illuminant the limiting curve in the foremost vertical plane will be the luminosity curve of the illuminant.

One of the points which is emphasized in dealing with colored media in the foregoing manner is that the spectral transmission and reflection factors are never zero, but are merely relatively low for some wave-lengths as compared with others. This is often forgotten when spectral analyses are made with instru- 
ments, because when the luminosity falls below the threshold the transmission factor is considered to be zero; however, the threshold depends upon the intensity of illumination or upon the brightness of the light-source.

\section{SPECTRAL ANALYSES OF GLASSES.}

In the development of colored glasses for the variety of practical applications the spectral analyses are extremely valuable and often essential. By means of such data these coloring elements can be mixed computationally to obtain the desired spectral characteristic. From very meagre data on the chemical composition from one melt fairly definite strides toward realization may be made in succeeding melts. Of course, there are chemical consiclerations which sometimes alter the predictions based on computation; however, such a procedure forms a most definite working basis. In the combination of glasses for special filters, lighting effects, etc., the computational method often saves time and provides definite data. Sometimes only the subjective color is desired, but even in these cases spectral analyses of elemental colorings provide the basis for manipulating the available vitrifiable colored media in a manner analogous to the combination of pigments.

In the manufacture of colored glass there is a limited number of coloring materials available, and when the glass must be limited to one general composition, such as soda lime, for example, the colors which are possible of attainment are further limited. However, by combining various coloring materials the variety of colored glasses can be enormously extended to meet the requirements of science and art.

In this paper the spectral analyses of a few fundamental colored glasses will be presented, and also the results of a few simple combinations. The record number of the specimen is placed before the symbol of the coloring metal, such as 37 Se. If different relative thicknesses of the specimen are presented, a number is placed before the designation proper, as ro (37 Se) indicates ro units of thickness (or of concentration), CS indicates lime soda glass, PS lead soda, BS barium soda, P lead, etc. Red.-Selenium, copper, and gold are commonly used for producing red glasses. In Fig. I 3 are shown the spectral analyses of a number of selenium glasses. It is seen that some of these are yellow in appearance and vary from this to a deep red. The 
composition of the mix is sometimes of considerable influence upon the final color. Specimen I4 Se, shown in relative thickness, IO, 20, and 34, was of unknown composition, but the coloring element was selenium. This is a remarkable specimen. Cobalt blue glass (Fig. I7) transmits a deep red band, so a combination of dense cobalt blue and selenium glasses isolates a deep-red band, as seen in $6 \mathrm{Co}+\mathrm{I}_{4} \mathrm{Se}$, Fig. I 3 .

By computations similar to those presented in the case of pigments (substituting transmission factors, $T_{\lambda}$, for reflection factors $R_{\lambda}$ ) the efficiency of such a combination in transmitting

FIG. I3.



only a deep-red band can be compared with that of a very dence selenium, gold, or copper glass. Unfortunately, at the ends of the visible spectrum the visibility data are difficult of determination and have been the least investigated; however, Hyde, Cady, and Forsythe ${ }^{9}$ have determined the visibility at the extreme red end of the visible spectrum with great care.

In Fig. 14 is shown the spectral characteristic of a copper-red glass, ${ }_{4} \mathrm{Cu}$, and in Fig. I 5 the spectral analyses of gold glasses are presented. Gold produces a beautiful pink in low concentrations (or in thin layers) and deep red in the higher ones (or

${ }^{\circ}$ Astrophysical Joumal, 42, p. 285 , 1915. 
FIG. I4.

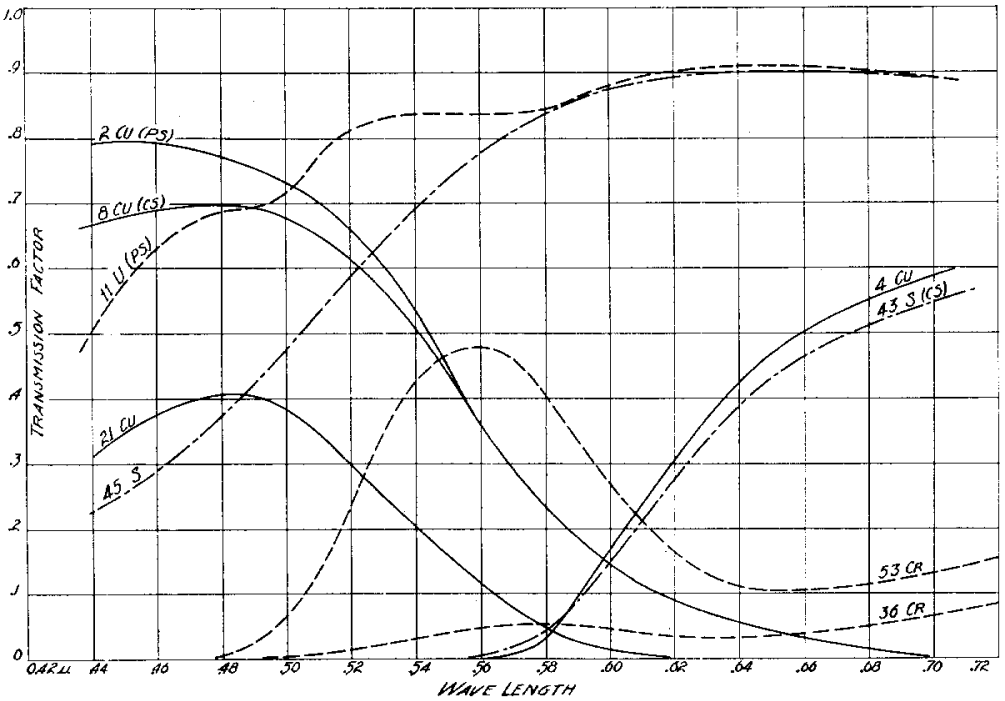

Spectral transmission-factors of copper, sulphur, chromium, and uranium glasses.

FIG. I5.

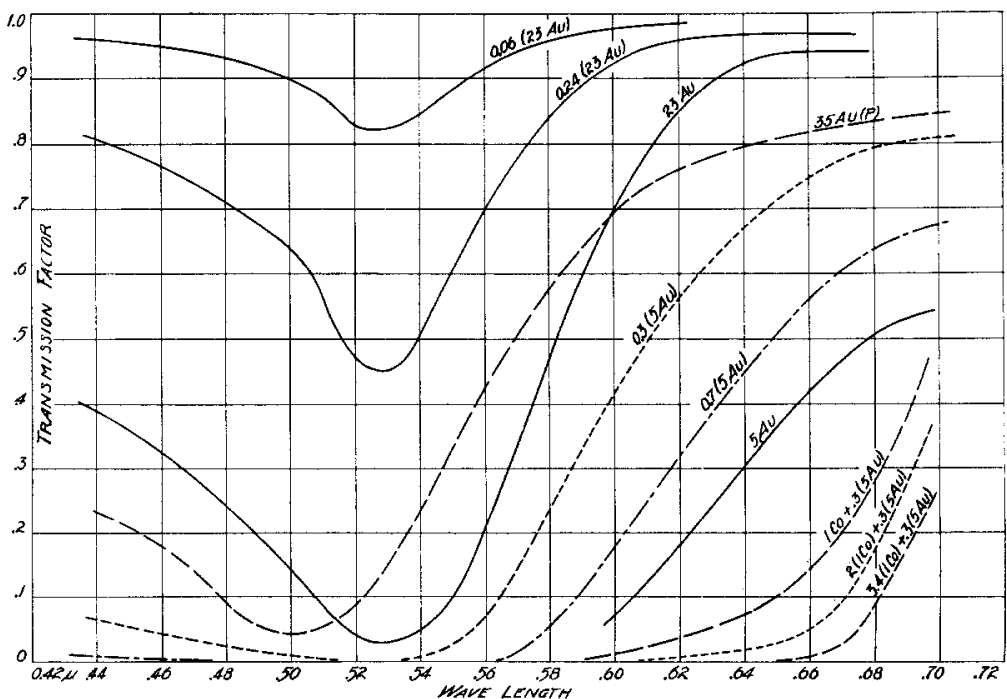

Spectral transmission-factors of gold glasses and combinations of cobalt. 
in thicker layers). The absorption band is seen to be near $0.53^{\mu}$ for the more transparent glasses, and it is interesting to note glass $35 \mathrm{Au}$, a lead gold, which shows a shift in the absorption band to $0.50 \%$. This glass was reheated several times in bringing out the color, which is decidedly more ruddy, and it appears that there is a different state of division of the metallic particles, perhaps, as to size. As the concentration or thickness increases (glass $5 \mathrm{Au}$, which is shown for three thicknesses) the blue band gradually disappears; however, the transmission does not closely approach monochromatism. In Fig. 15 are also shown FIG. 16.

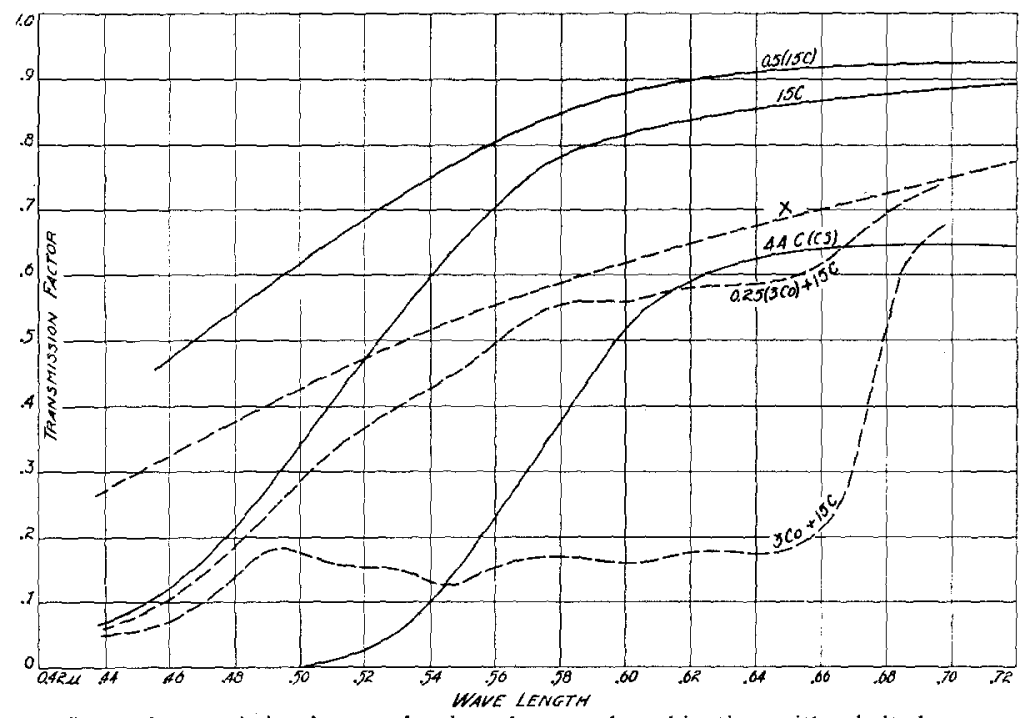

Spectral transmission-factors of carbon glasses and combinations with cobalt glasses.

the results of combining cobalt and gold glasses of different thicknesses (or concentrations), with the resulting transmission confined to the deep-red region.

Yellow-Carbon, sulphur, uranium, and silver are among. those elements which, when introduced into glass under proper chemical conditions, produce yellow glasses of varying color. No single element isolates spectral yellow. In Fig. 16 are shown the spectral analyses of carbon yellow glasses, $5_{5} \mathrm{C}$ and $44 \mathrm{C}$, and of combinations of carbon yellow and light cobalt blue glasses. It is known that $\mathrm{X}$-rays, ultraviolet and visible rays will cause some clear glasses to become colored. In Fig. 16 is also shown the 
spectral characteristic of a glass $X$, which, though originally clear, was colored a muddy yellow throughout the mass by X-rays. It is interesting to observe the action of X-rays in discoloring glass, for it is easy at times to observe the progress of the coloring through the thickness of the glass. Patterns can be made by this process. In Fig. I4 are shown the spectral characteristics of uranium ( I I U) and sulphur ( $43 \mathrm{~S}$ and $45 \mathrm{~S}$ ) glasses. The spectral transmissions of several uranium samples appear to be kinky in the blue-green region, although the exact nature of the curves are not established.

Grecn.-Iron imparts a green color to glass, varying from a bluish to a yellowish-green, depending upon the ingredients of the glass. The importance of manganese in glass is as a decolorizing agent, its color in proper concentrations being roughly complementary to that of iron commonly present in sand. Chromium imprarts a yellowish-green color to glass, as seen in glass $53 \mathrm{Cr}$, Fig. I 4. This glass has a maximum transmission at about $0.56 \mu$, and by the addition of copper blue-green (glasses $2 \mathrm{Cu}$ and $8 \mathrm{Cu}$, Fig. I4) this maximum can be shifted toward the shorter wavelengths, depending upon the proportions of the coloring elements. Glass 2 I $\mathrm{Cu}$, called signal blue-green, is evidently a copper glass. Glass $36 \mathrm{Cr}$ is a dense chromium green. In order to compare the actual colors under a given illuminant it is well to reduce these curves to luminosity values. If monochromatism is desired, it is often advisable to combine two glasses which transmit a narrow region in common.

Blue. - Cobalt is the most common element used to impart a blue color to glass. Its greatest disadvantage (although sometimes an advantage) is its transmission of a deep-red band, as shown in $6 \mathrm{Co}$ and $7 \mathrm{Co}$, Fig. $\mathrm{I} 7$. This red transmission can be utilized in isolating the deep red, as shown by combining cobalt and selenium or other red glasses, for example I $\mathrm{Co}+\mathrm{I}_{4} \mathrm{Se}$. An excellent blue glass can be made by combining cobalt with copper blue-green, for the latter effectively absorbs the deep red. The spectral characteristic of such a combination is shown in $9 \mathrm{Cu}+$ $6 \mathrm{Co}$, Fig. I 7.

Purple.-Nickel produces a purple color in glass, and also manganese, but the latter is not an efficient purple, because its absorption band is not sharp. Its chief use is to neutralize the green tint due to the presence of iron in the ingredients of glass 
FIG. 17.

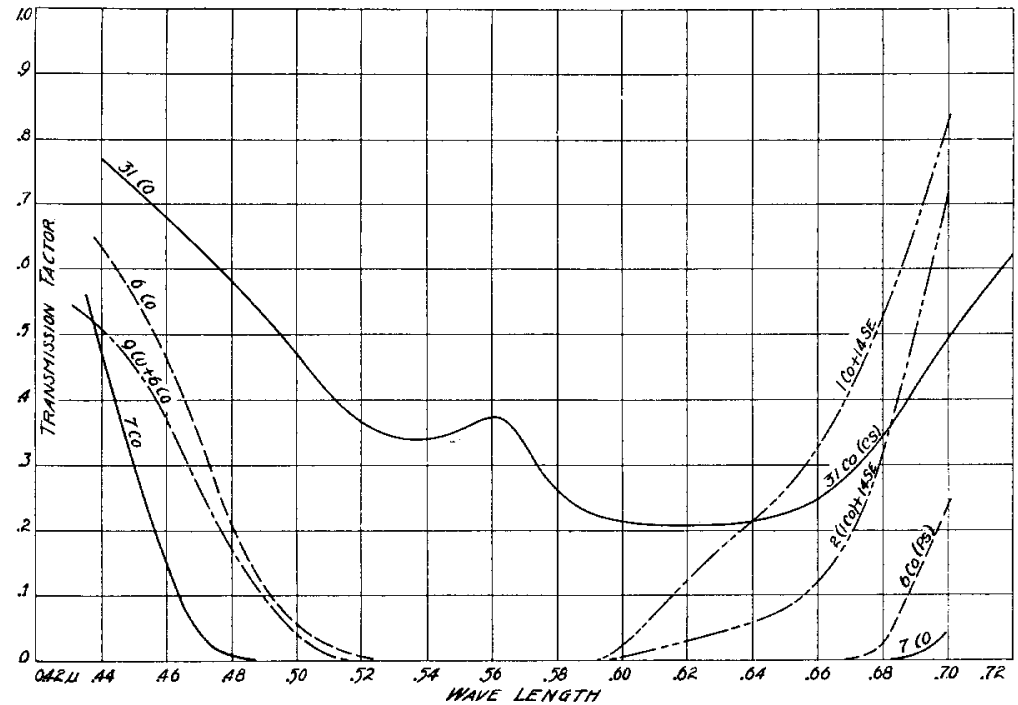

Spectral transmission-factors of cobalt glasses.

FIG. 18.

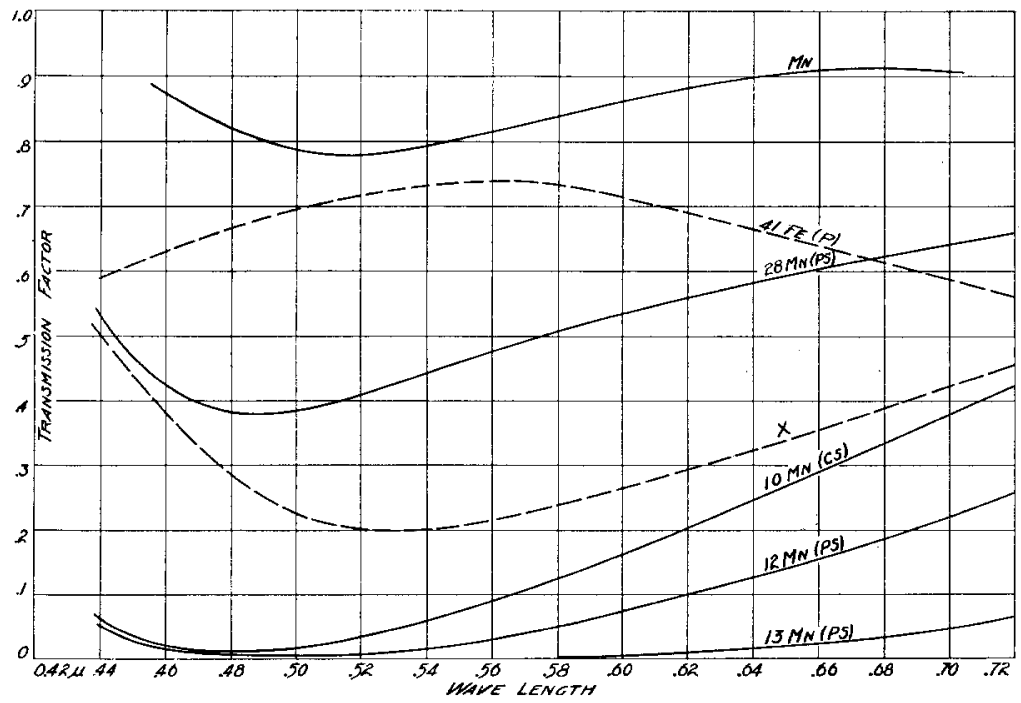

Spectral transmission-factors of manganese and iron glasses. 
mixes. The spectral characteristic of a glass containing iron is shown in $4 \mathrm{I} \mathrm{Fe}$, Fig. I8. It is seen that a manganese glass of proper density is approximately complementary in color to the iron glass. Although the manganese neutralizes the iron in color, the transmission factor of the resultant glass may be seriously reduced. Manganese, though a useful element in glass manufacture, cannot be considered important as a coloring element from the viewpoint of colored glass in general. In $X$, Fig. I 8 , is shown the spectral characteristic of an originally clear glass which has been colored a deep-purplish hue by exposure to X-rays. Undoubtedly this coloring is due to an effect upon the manganese present in the clear glass. This effect is commonly observed in lamp globes and window glass exposed to strong sunlight. In the former cases it is a very serious defect of glass manufacture, because the author has observed such globes whose transmission has been reduced as much as $5^{\circ}$ per cent. after long exposure to intense solar radiation or to that emitted by an arc lamp. It would be far better in such cases as street-lighting glassware to eliminate the manganese and to endure the unneutralized greenish hue of the iron which is mavoidably present.

USES OF SPECTRAL ANALYSES OF GLASSES.

The applications for spectral analyses of colored glasses have been fairly well covered in the discussions of pigmenıs and dyes. for the same general procedures can be applied to colored glasses. The concentration is not so definite as in the case of dyes, because, owing to the high temperature at which glass melts and to chemical action, the concentration of coloring material in the final glass cannot always be predicted from the amount of the coloring metal added to the mix. Some of the metals, such as cobalt and copper, under standardized conditions of melting, appear to produce concentrations of coloring material proportional to the amounts of the oxides added to the mix, but in some cases there is doubt as to this proportionality. There is need for systematic study in this direction. In the case of the red glasses, for example gold ruby, which in ordinary manufacture assumes its red color on reheating, the manipulation has considerable effect upon the density of the color. After a colored glass has been obtained it is possible to procure from a single spectral analysis the integral transmission factor for any illuminant, the spectral characteristics of other thicknesses, and those of combinations of these thicknesses with other colored glasses as already ontlined. 
For the sake of further exemplification, in Fig. I9 are shown the straight-line relations between thickness and transmission factor (for entering radiation) for several wave-lengths for various thicknesses of a gold ruby glass. The relations between luminosity and thickness for this glass are shown in Fig. 20 for various wave-lengths. Fig. I9 is a diagram of what would be seen if the solid represented in Fig. I 2 were viewed from the right-hand side.

At this point it is of interest to show the approximation of experimental results to the relations between spectral character and thickness as predicted by theory. This is shown in Fig. $2 \mathrm{I}$

FIG. I9.

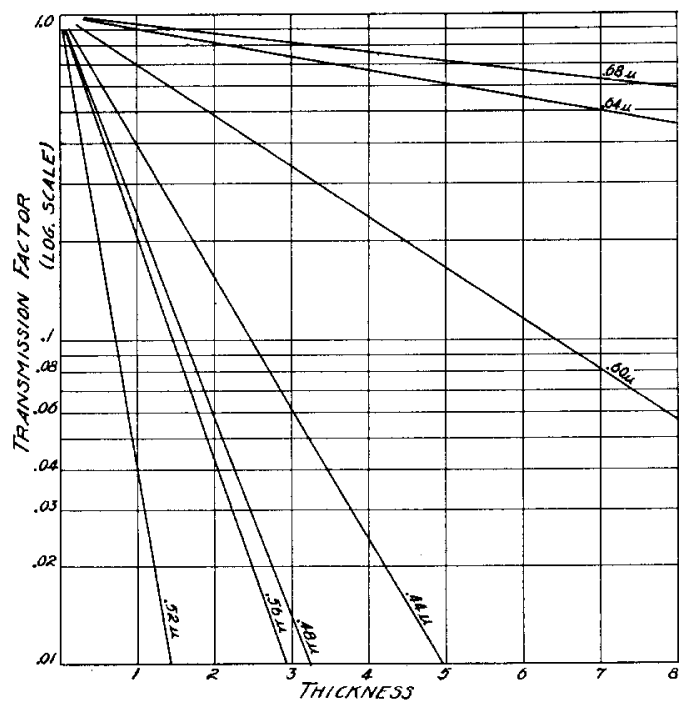

Relations between spectral transmission-factor and thickness of a gold glass (23 $\mathrm{Au}$ ).

for one of the glasses made during the development of an "artificial-daylight" glass some years ago. The method of graphical analysis was tested because of the desire for a simple method, and the specimen was ground and polished in five thicknesses. The circles show the verification of the theory. In this case correction had not been made for surface reflection, so the straight lines must be drawn to a point near 0.92 on the transmission axis. Incidentally it is of interest to note that, previous to the adoption of this method, samples of melts were ground in the form of thin wedges and spectral analyses were made at various thicknesses. It is seen that the graphical method enormously reduces 
the amount of work in order to obtain the data necessary for such studies.

In developing a colored glass for a specific purpose various factors are considered, such as the illuminant to be used and the result to be obtained. From these an ideal spectral transmission curve is determined, and by means of a few spectral analyses of different colored glasses, bearing in mind the chemical consider-

FIG. 20.



Relations between spectral luminosity and thickness of a gold glass ( $23 \mathrm{Au}$ ).

ations if a mixture is finally necessary, various combinations can be made with the aid of the graphical method.

Often the ultraviolet and infra-red spectral transmissions are of interest, and these are made in the manner already described. The data on a coloring element are not considered to be sufficiently complete for record if the ultraviolet transmission is not studied at least qualitatively, and in some cases the infra-red 
transmission is investigated. It appears unnecessary to discuss these further, for the aim of this paper has been realized. Various data have been presented with the hope that they will be helpful to others, and various uses of physical data have been discussed with a view to presenting only aspects that are uncommonly appreciated and which have been developed in practical work in color-technology.

\section{SUMMARY.}

There have been presented in the foregoing:

A discussion of methods of analyzing color.

A discussion of general characteristics of pigments, dyes, and colored glasses.

FIG. 2 I.

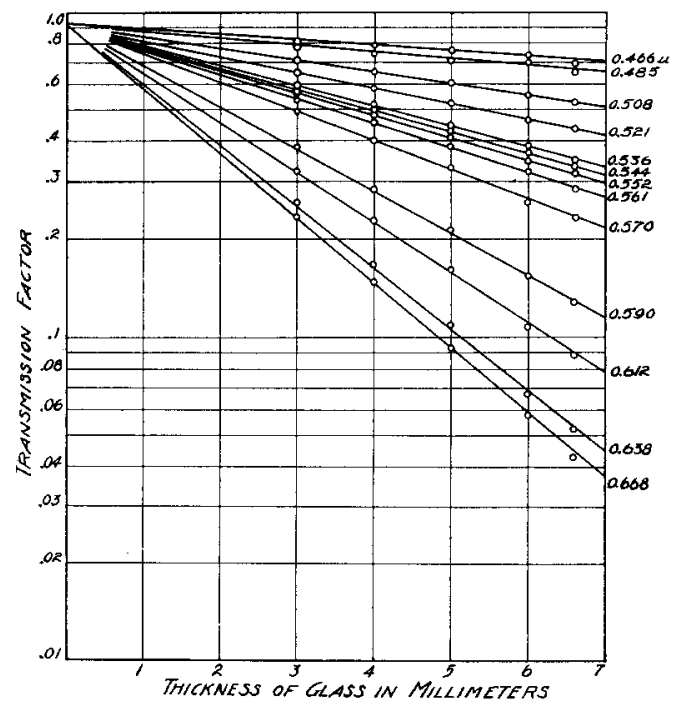

Test of the relation between spectral transmission-factor and thickness of a blue-green glass.

Laws of absorption and their uses.

Spectral analyses of the visible radiation reflected by pigments and transmitted by dye-solutions and colored glasses.

A discussion of the uses of such data.

A general emphasis of the importance of a physical basis in color-technology.

I wish to acknowledge the assistance of Mr. H. H. Kirby in obtaining the data and preparing the illustrations.

Cleveland, Ohio,

April 28, I9I7. 